\title{
An RF Wireless Power Transfer system to power battery-free devices for asset tracking
}

\author{
Roberto La Rosa*, Catherine Dehollain ${ }^{\dagger}$, Filippo Pellitteri ${ }^{\ddagger}$, Rosario Miceli ${ }^{\ddagger}$, Patrizia Livreri ${ }^{\ddagger}$ \\ * STMicroelectronis Catania, Italy \\ Email: roberto.larosa@st.com \\ $\dagger$ Ecole Polytechnique Federale de Lausanne, Switzerland \\ $\ddagger$ Department of Engineering, University of Palermo, Italy
}

\begin{abstract}
Internet of Things (IoT) and Wireless Sensor Networks (WSN) infrastructures are becoming more and more available and diffused. One major outcome is the development of new services that help to make everyday life easier and better. One of those to which this paper reserves special attention is asset tracking which refers to the method of tracking physical assets. This service is very well based on IoT infrastructure and, due to the enormous number of objects to be traced, desperately needs the availability of inexpensive tags with sensing capabilities, that can be conveniently monitored from a long distance and require no maintenance. For this, engineers are called to face very challenging issues. One of these is how to power a maintenance-free tag that consequently must be battery-free. This paper deals with the investigation of a system based on radio frequency (RF) Wireless Power Transfer (WPT) that powers a battery-free tag for asset tracking, in which the major challenge is that the WPT deals with dynamic objects at different speeds. The performances and key features of a system on chip (SoC) specifically designed to power a tag by means of RF WPT will be investigated. The aim is to provide a strategy and a model to design the infrastructure by providing the number of hot-spots needed to perform successfully the identification of an asset. Validation of the model has been done by means of specific tests and experimental results are provided.
\end{abstract}

Index Terms-WSNs, Radio Frequency, Energy Harvesting, Wireless Battery Charger, Lithium Ion, Battery, Wireless Sensor Networks, Internet of Things

\section{INTRODUCTION}

The very promising market of Internet of Things (IoT) devices is leading to smart systems and advanced services. Those innovative solutions require embedded communication and sensing capabilities, signal processing, power management and power generation. The main evolution so far is represented by Wireless Sensor Networks (WSN).

As a consequence, the wireless node has evolved from a simple tag transponder mostly used for short distance item identification to a more complex device able to perform sensing, data analysis and communication. These extra performances require more energy, which in many cases is provided by a battery which inevitably poses a problem of costs, maintenance and miniaturization. In very common working conditions [1] a significant reduction in battery power consumption can be achieved by reducing or eliminating standby power [2]-[6] that directly translates into higher system lifetime or system miniaturization. Unfortunately, this solution can alleviate maintenance and miniaturization problems but it does not really solve them. For this reason, where possible, such as in low duty-cycle sensors, it is by far preferred to implement battery-free devices, with the evident advantage to render them not-disposable, with virtually unlimited lifetime and more cost efficient [7]. For this reason, batteries are normally avoided and this leads engineers to choose, as power source, primarily RF Wireless Power Transfer (WPT) and where possible even radio-frequency (RF) Energy Harvesting (EH) [8]-[11]. Except for the case of a few centimeters distance between the power source and the supplied device [12], [13], efficient WPT and RF EH is not a simple task [14]. In fact, while RF energy has the advantage to be pervasive, so that it can be conveniently transferred to out-of-sight places, on the other side the efficiency of the power transfer is very low [15].

All this suggests that engineers need to take a lot of care in the design of the various parameters and devices involved in WPT systems, such as the RF to DC converter, the power transmitter and the antennas [16]. Regarding the RF to DC converter, there are recent contributions in the design of ultra-low power integrated circuits with system on chip (SoC) that achieve a good level of integration and high performances both in terms of power conversion efficiency (PCE) and power sensitivity [17]-[21].

The aim of this paper is to study the RF WPT between a power transmitter (hot-spot) and a power receiver (a battery-free tag) in a typical asset tracking work environment where the assets travel at a certain speed related to the hot-spots. The peculiarity of this study is to evaluate WPT in a dynamic environment where the distance between the power transmitter and the RF to DC converter cannot be considered fixed as they move related to each other at a defined speed. This study case has been considered to identify the conditions in order to allow the continuous tracking of a moving asset. In particular, the focus of this investigation is to estimate the minimum number of required hot-spots to continuously power a moving tag. The paper is organized as follows. Section II describes the system in terms of antennas, power transmitter, $\mathrm{RF}$ to DC converter and the asset tag. It also introduces the SoC used to implement the RF to DC converter. Section III shows the static characterization of the proposed RF WPT 
system, in terms of current at the output of the RF to DC converter as a function of the distance from the RF power transmitter. Section IV describes the dynamic characterization of the RF WPT through both simulation and experimental tests. Sections V reports the final conclusions.

\section{SYSTEM DESCRIPTION}

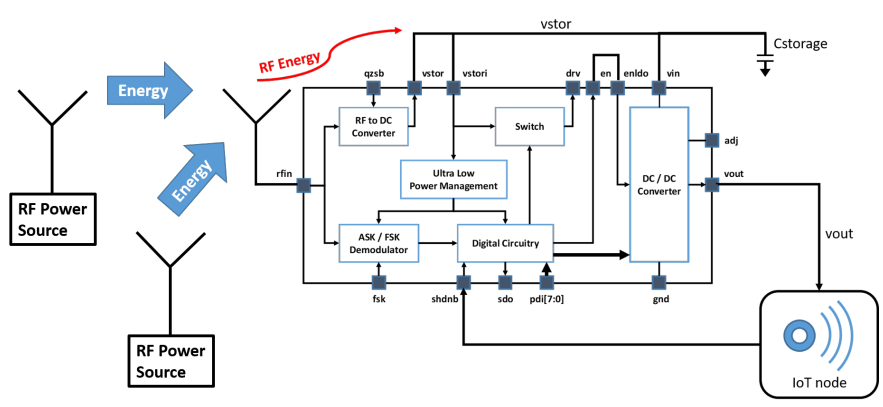

Fig. 1. Wireless Power Transfer system description

As shown in Fig. 1, the system consists of one or more power transmitters and a SoC as $\mathrm{RF}$ to $\mathrm{DC}$ converter that provides power to a generic asset tag. In this paper, a generic asset tag with an energy requirement of 0.4 mJoule, which corresponds to an average current consumption of $4 \mathrm{~mA}$, an acquisition time of $50 \mathrm{msec}$ and a bias voltage of $2 \mathrm{~V}$, will be considered, consisting of the integration of a low power microcontroller, humidity and temperature sensor and Bluetooth Low Energy radio. It is implemented to be a battery-free device as it is powered by means of WPT with a SoC that performs the function of RF to DC converter. The power receiver platform is a $2.5 \mu \mathrm{W}$ SoC which integrates a wide-bandwidth (350 MHz-2.4 GHz) RF to DC power converter with an input power sensitivity as low as $-18.8 \mathrm{dBm}$ and a maximum PCE of $45 \%$ at $900 \mathrm{MHz}$ with an input power of $-10 \mathrm{dBm}$ and the output voltage of $2.2 \mathrm{~V}$. The ultra-low power management is essentially a nano-power circuit with a quiescent current as low as $75 \mathrm{nA}$ that allows scavenging power as low as $\mu \mathrm{W}$.

\section{STATIC CHARACTERIZATION OF THE RF TO DC CONVERTER}

\section{A. Experimental measurements}

In order to characterize the SoC, at the frequency of $900 \mathrm{MHz}$, in terms of current at the output of the RF to DC converter as a function of the distance from an RF power source, a set of measurements has been carried out inside a semi-anechoic chamber, as shown in Fig. 2, where all the surfaces are absorbing, except for the pavement which is reflective. Each set consisted of different measurements of $\mathrm{V}$ (vstor), which is the voltage across the storage capacitor $\mathrm{C}_{\text {storage, }}$ at different distances between an RF power source, also called "hot-spot", and the SoC, also called "tag".

According to the time needed to charge $\mathrm{C}_{\text {storage }}$ at the WPT start-up, the current at the output of the RF to DC converter has been characterized for each investigated value of distance.
Each measurement has been carried out in static conditions, without mutual movement between hot-spot and tag. As any capacitance current, the measured current is therefore linked to the time in which $\mathrm{V}$ (vstor) rises from $0 \mathrm{~V}$ to the $2.2 \mathrm{~V}$ maximum value, considering the capacitance $\mathrm{C}_{\text {storage }}$, equal to $990 \mu \mathrm{F}$ to store a 0.4 mjoule with a voltage that varies from $2.2 \mathrm{~V}$ down to $2.0 \mathrm{~V}$. Both the power transmitter and the RF energy harvester are equipped with the Revie Pro antenna provided by Laird [22]. A model of the current vs distance behaviour has been therefore obtained and electrically simulated in order to foresee the system response in case of dynamic variation of the distance between the hot-spot and the tag.

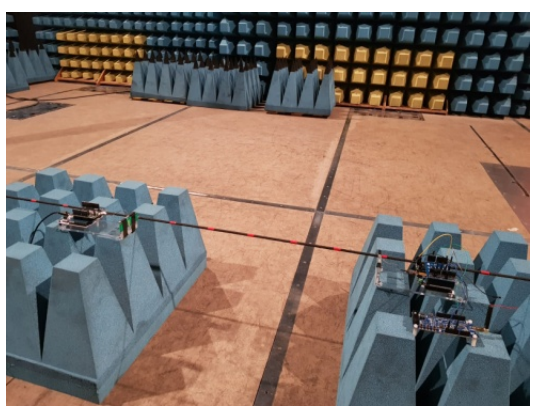

Fig. 2. Experimental setup: the hot-spot is on the left, the tag is on the right

\section{B. Equivalent model of the RF to DC converter}

The experimentally obtained results, at the frequency of $900 \mathrm{MHz}$, are represented by the black spots reported in Fig. 3. where the current I, at the output of the RF-to-DC converter, is given as a function of the distance $d$. The blue curve implements a mathematical approximation (represented by a 5th degree polynomial) used to cover the entire range of distance values, since these measurements have been done only at discrete values of distance, and therefore it is useful to provide a model. The non-monotonic evolution is due to the non-linear behaviour of the PCE of the RF to DC converter.

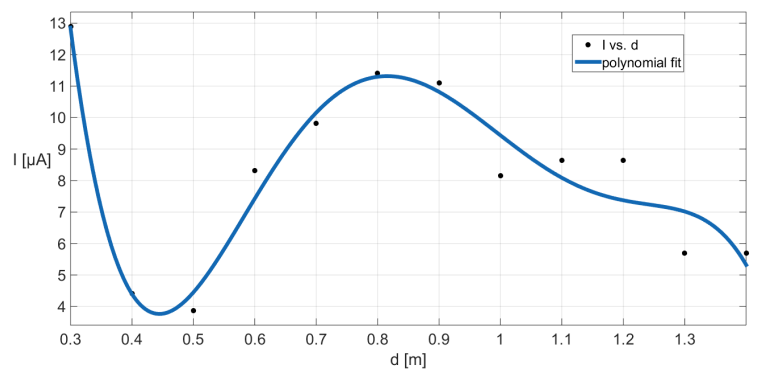

Fig. 3. Polynomial fit of the current vs. distance plot

The aim of this mathematical elaboration, implemented through the software MATLAB, is to insert this model into the electrical model of the system, in order to foresee the behaviour of the capacitor voltage in case of dynamic WPT. 
The polynomial equation expressing the current I as function of the distance $\mathrm{d}$ is the following:

$$
I=p_{1} d^{5}+p_{2} d^{4}+p_{3} d^{3}+p_{4} d^{2}+p_{5} d+p_{6}
$$

where the coefficients have the following values:

$$
\begin{array}{rrrr}
p_{1}= & -0.00453 & p_{4}= & 0.03254 \\
p_{2}= & 0.02128 & p_{5}= & -0.01283 \\
p_{3}= & -0.03828 & p_{6}= & 0.00192
\end{array}
$$

\section{DYNAMIC CHARACTERIZATION}

\section{A. Simulation results}

In order to evaluate the case of a dynamic WPT, the electrical model of the system has been simulated according to a proper variation of the distance over time. A rotating circular platform holds the hot-spot, whereas the tag is placed on a fixed spot, out of the platform. In this case the hot-spot is in movement while the tag is fixed, but it is equivalent to the reverse case which is typical of asset tracking (tag in movement and fixed hot-spot), since the mutual position between them evolves as well. In Fig. 4 the geometrical image of the setup is highlighted. The tag is represented by $\mathrm{T}$, whereas the hot-spot by $\mathrm{H}$. It is supposed that $\mathrm{H}$ moves on the superior semi-circle alternatively clockwise (continuous arrow line) and anticlockwise (dashed arrow line). The movement in the inferior semi-circle is neglected since there the hot-spot is considered out-of-sight with respect to the tag, as highlighted from the shadowed part of the figure. The angle between the radius crossing the hot-spot and the radius $r$ is $\alpha$, whereas the distance between the hot-spot and the tag is d. In the figure, two possible positions of $\mathrm{H}$ are associated to the distances $\mathrm{d}_{1}$ and $\mathrm{d}_{2}$ and to the angles $\alpha_{1}$ and $\alpha_{2}$.

The distance $\mathrm{d}$ is therefore obtained as follows:

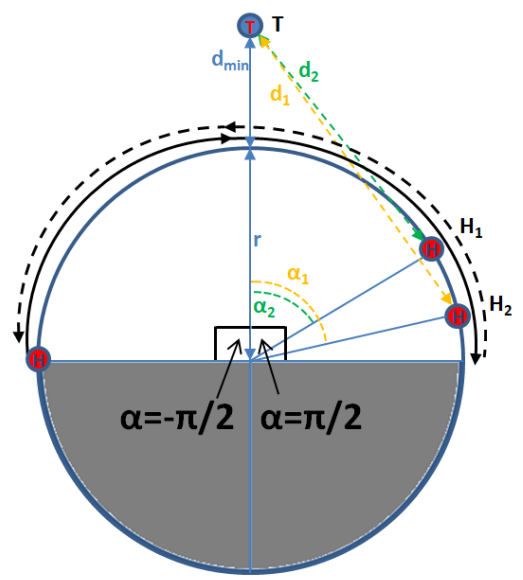

Fig. 4. Geometrical representation of the rotating platform

$$
d=\sqrt{(r \sin \alpha)^{2}+\left(d_{\min }+r-r \cos \alpha\right)^{2}}
$$

where the angle $\alpha$ ranges between $-\pi / 2$ and $\pi / 2$.
The angle $\alpha$ is related to the rotational speed of the platform $\omega$ and to the time $t$ as follows:

$$
\alpha=\omega t
$$

In Fig. 5 the capacitor voltage V(vstor) evolution at the cold start phase is simulated along with the current I at the RF to DC converter output, through the software LTSpice, according to the variation of the distance over time, as described by (3) and (4), considering that the hot-spot moves at the speed of $0.1 \mathrm{~m} / \mathrm{s}$ across an average distance of 0.85 meters passing in front of the tag which is placed at the fixed minimum distance of 0.3 meters. As soon as the voltage level reaches the maximum value of $2.2 \mathrm{~V}$, the SoC circuitry switches alternatively from the charge to the active phase and the $\mathrm{V}$ (vstor) begins to fluctuate between two thresholds.

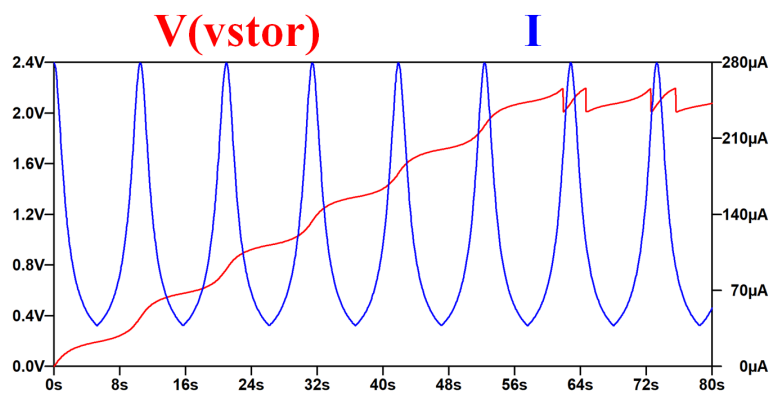

Fig. 5. Evolution of the simulated voltage (in red) and current (in blue) at the RF to DC output in case of dynamic WPT

If the reverse situation is imagined, that is one moving tag and a number $\mathrm{n}$ of fixed hot-spots, the aim of this investigation is provide a simple model to derive the minimum number of hot-spots that guarantees the power up of the moving tag during the cold start phase. This is useful to give a design approach for a continuous tracking of assets which are provided with battery-free sensor nodes. Considering Fig. 5 , each step of V(vstor) at the start-up of the dynamic WPT is the equivalent of one fixed hot-spot. The minimum number of required hot-spots would be therefore 7 in this case.

\section{B. Experimental results}

In Fig. 6 the tested V(vstor) is shown. The hot-spot moves with the platform at $0.1 \mathrm{~m} / \mathrm{s}$.

A minimum number $\mathrm{n}$ of 7 required hot-spots is confirmed. In Fig. 7 an alternative experimental setup for the dynamic characterization of the RF WPT system is shown. This setup consists of a fixed tag, placed atop a tripod, and a number of hot-spots, placed on a conveyor belt, at the mutal distance of 1 meter, and moving at the aforementioned speed, of $0.1 \mathrm{~m} / \mathrm{s}$. A similar storage capacitor voltage has been tested.

\section{Conclusions}

In this paper, a system based on RF WPT to power a battery-free tag for asset tracking has been investigated. The most challenging requirement is to make this WPT able to cope with the dynamic movements of the tags. In order to 


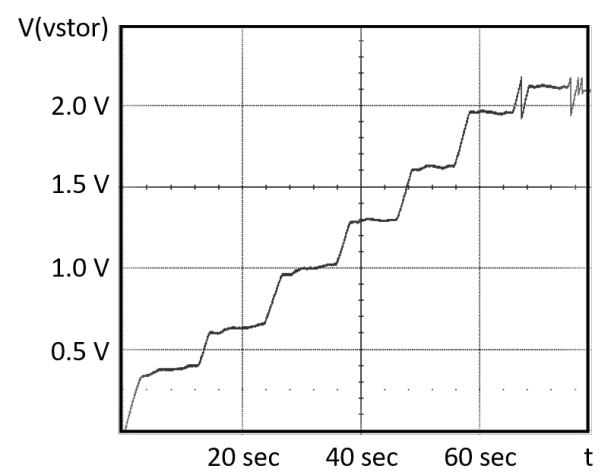

Fig. 6. Measured storage capacitor voltage as a function of the time in case of dynamic WPT: time division $=20 \mathrm{~s} / \mathrm{div} ;$ Volt division $=0.5 \mathrm{~V} / \mathrm{div}$
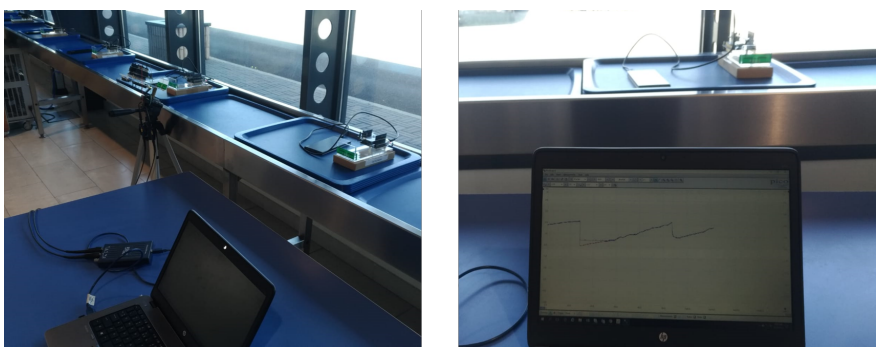

Fig. 7. Setup and wave-forms of tests performed on the conveyor belt

solve this issue the following steps have been performed. The proposed SoC has been characterized in order to derive a proper design model able to estimate the minimum number of fixed hot-spots to guarantee a continuous power supply of a moving tag during its cold start phase. An analytical and a SPICE model have been derived to forecast the minimum number of hot-spots to successfully supply the power to a tag during its cold start phase. The validation of the model has been performed through specific experimental tests both in the semi-anechoic chamber employing a rotating platform and on field environment conditions employing a conveyor belt. The experimental results have proven to be in agreement with the models and confirm, for the given conditions of speed and distance between the power transmitter and RF to DC converter, the minimum requirement of 7 hot-spots to supply an asset tracking tag.

\section{REFERENCES}

[1] A. Yamawaki and S. Serikawa, "Battery life estimation of sensor node with zero standby power consumption," in 2016 IEEE Intl Conference on Computational Science and Engineering (CSE) and IEEE Intl Conference on Embedded and Ubiquitous Computing (EUC) and 15th Int Symposium on Distributed Computing and Applications for Business Engineering (DCABES), 2016, pp. 166-172.

[2] R. La Rosa, N. Aiello, and G. Zoppi, "An innovative system capable to turn on any turned off electrical appliance by means of an efficient optical energy transfer," in PCIM Europe 2014; International Exhibition and Conference for Power Electronics, Intelligent Motion, Renewable Energy and Energy Management. VDE, 2014, pp. 1559-1566.

[3] — , "RF remotely-powered integrated system to nullify standby power consumption in electrical appliances," in Industrial Electronics Society, IECON 2016-42nd Annual Conference of the IEEE. IEEE, 2016, pp. $1162-1164$.
[4] C. Trigona, B. Andò, S. Baglio, R. La Rosa, and G. Zoppi, "Vibrationbased transducer for zero-energy standby applications," in Sensors Applications Symposium (SAS), 2016 IEEE. IEEE, 2016, pp. 1-4.

[5] - "Sensors for kinetic energy measurement operating on zerocurrent standby," IEEE Transactions on Instrumentation and Measurement, vol. 66, no. 4, pp. 812-820, 2017.

[6] D. Gerber, A. Meier, R. Hosbach, and R. Liou, "Zero standby solutions with optical energy harvesting from a laser pointer," Electronics, vol. 7, no. 11, p. 292, 2018.

[7] R. La Rosa, C. Trigona, G. Zoppi, C. Di Carlo, L. Di Donato, and G. Sorbello, "RF energy scavenger for battery-free wireless sensor nodes," in 2018 IEEE International Instrumentation and Measurement Technology Conference (I2MTC). IEEE, 2018, pp. 1-5.

[8] O. Kazanc, J. A. Rodríguez-Rodríguez, M. Delgado-Restitute, F. Maloberti, and C. Dehollain, "Far-field uhf remotely powered front-end for patient monitoring with wearable antenna," in 2013 IEEE 11th International New Circuits and Systems Conference (NEWCAS). Ieee, 2013, pp. 1-4.

[9] K. Kapucu and C. Dehollain, "A passive uhf rfid platform for sensing applications," in 2015 6th International Workshop on Advances in Sensors and Interfaces (IWASI). IEEE, 2015, pp. 146-151.

[10] O. Kazanc, F. Maloberti, and C. Dehollain, "Remotely-powered frontend at $2.45 \mathrm{ghz}$ for real-time continuous temperature sensing," in 2018 Ieee International Conference On Rfid (Rfid). IEEE, 2018, pp. 1-7.

[11] N. Pillin, N. Joehl, C. Dehollain, and M. J. Declercq, "Wireless voltage regulation for passive transponders using an if to communicate," IEEE Transactions on Circuits and Systems I: Regular Papers, vol. 57, no. 3, pp. 714-724, 2009.

[12] V. Castiglia, L. Di Noia, P. Livreri, R. Miceli, C. Nevoloso, F. Pellitteri, and F. Viola, "An efficient wireless power transfer prototype for electrical vehicles," in 2017 IEEE 6th International Conference on Renewable Energy Research and Applications (ICRERA). IEEE, 2017, pp. 12151220 .

[13] V. Boscaino, F. Pellitteri, L. Rosa, and G. Capponi, "Wireless battery chargers for portable applications: design and test of a high-efficiency power receiver," IET Power Electronics, vol. 6, no. 1, pp. 20-29, 2013.

[14] K. Kapucu, J. L. M. Panades, and C. Dehollain, "Design of a passive uhf rfid tag for capacitive sensor applications," in Proceedings of the 2013 9th Conference on Ph. D. Research in Microelectronics and Electronics (PRIME). Ieee, 2013, pp. 213-216.

[15] R. L. Rosa, G. Zoppi, A. Finocchiaro, G. Papotto, L. D. Donato, G. Sorbello, F. Bellomo, C. A. D. Carlo, and P. Livreri, "An over-thedistance wireless battery charger based on RF energy harvesting," in 2017 14th International Conference on Synthesis, Modeling, Analysis and Simulation Methods and Applications to Circuit Design (SMACD). IEEE, 2017, pp. 1-4.

[16] C. Di Carlo, L. Di Donato, G. Mauro, R. La Rosa, P. Livreri, and G. Sorbello, "A circularly polarized wideband high gain patch antenna for wireless power transfer," Microwave and Optical Technology Letters, vol. 60 , no. 3, pp. 620-625, 2018.

[17] R. La Rosa, P. Livreri, C. Trigona, L. Di Donato, and G. Sorbello, "Strategies and techniques for powering wireless sensor nodes through energy harvesting and wireless power transfer,' Sensors, vol. 19, no. 12, 2019. [Online]. Available: https://www.mdpi.com/1424-8220/ $19 / 12 / 2660$

[18] R. Guerra, A. Finocchiaro, G. Papotto, B. Messina, L. Grasso, R. La Rosa, G. Zoppi, G. Notarangelo, and G. Palmisano, "An RFpowered fsk/ask receiver for remotely controlled systems," in Radio Frequency Integrated Circuits Symposium (RFIC), 2016 IEEE. IEEE, 2016, pp. 226-229.

[19] G. Papotto, N. Greco, A. Finocchiaro, R. Guerra, S. Leotta, and G. Palmisano, "An RF-powered transceiver exploiting sample and hold operation on the received carrier," IEEE Transactions on Microwave Theory and Techniques, vol. 66, no. 1, pp. 396-409, 2018.

[20] G. Papotto, F. Carrara, A. Finocchiaro, and G. Palmisano, "A 90nm cmos $5 \mathrm{mb} / \mathrm{s}$ crystal-less rf transceiver for rf-powered wsn nodes," in 2012 IEEE International Solid-State Circuits Conference. IEEE, 2012, pp. 452-454.

[21] X. Li, Y. Lu, M. Huang, and R. P. Martins, "A 2.4-ghz mid-field cmos wireless power receiver achieving $46 \%$ maximum pce and $163-\mathrm{mw}$ output power," IEEE Transactions on Circuits and Systems II: Express Briefs, 2019.

[22] "https://www.lairdtech.com/datasheet-revie-pro-0618pdf," 2018. 